\title{
Validación de un instrumento para medir conocimientos, percepción de riesgo y estigmas sobre tuberculosis
}

\author{
Validation of an instrument to measure knowledge, risk perception and \\ stigmata about tuberculosis
}
Karen Ahumada ${ }^{1}$, Lina Strba Idarraga ${ }^{1}$, Johana Alarcón Soto ${ }^{1}$, Jorge Figueroa Gómez $^{2,3}$, Yesenia Niño Ramírez ${ }^{3,4}$, Robinson Pacheco ${ }^{5}$
${ }^{1}$ Grupo de Investigación en Epidemiologia y Salud Pública, Universidad Libre, Cali ${ }^{2}$ Universidad Icesi, Departamento de Estudios Sociales y Estudios Políticos
${ }^{2}$ Universidad Icesi, Departamento de Estudios Sociales y Estudios Políticos
${ }^{3}$ Universidad Libre, Cali
${ }^{4}$ Secretaria de Salud Pública Municipal, Cali, Colombia
${ }^{5}$ Grupo de Investigación en Epidemiología y Servicios GRIEPIS, Universidad Libre, Cali

\section{Resumen}

Antecedente: La tuberculosis es una enfermedad infecciosa que suele afectar a los pulmones, causada por una bacteria (Mycobacterium tuberculosis). Se trasmite de una persona a otra a través de gotitas de aerosol. Esta enfermedad es una de las primeras causas de mortalidad a nivel mundial; por esto, es relevante validar un instrumento que nos permita evaluar conocimientos, percepciones de riesgo y estigmas sobre tuberculosis.

Objetivo: Diseñar y validar un instrumento para medir conocimientos, percepciones de riesgo y estigmas sobre tuberculosis en población privada de la libertad.

Metodología: Se realizó un estudio observacional descriptivo transversal. 11 expertos evaluaron de forma cualitativa y cuantitativa el instrumento preliminar. La evaluación cualitativa se realizó a través de un grupo focal. La evaluación cuantitativa de los ítems se realizó a través de escala tipo Likert, evaluando los siguientes criterios: Coherencia, relevancia y claridad. Y en cuanto a la evaluación de las dimensiones, esta se realizó por medio del criterio de suficiencia. Posterior a los ajustes se realizó la prueba piloto a 16 sujetos del centro penitenciario.

Resultados: De los 20 ítems propuestos inicialmente, el consenso de expertos ajustó la estructura, se elimina 4 ítems y adiciona un nuevo ítem, quedando el instrumento con 17 ítems. Se evaluó la validez del contenido a través del coeficiente V de Aiken 0,95 IC95\%

Fecha de envío: 27/03/2021

Fecha de aceptación: 30/03/2021 
$(0,82-0,99)$; como este salió perfecto, se evaluó la fiabilidad utilizando el Alfa de Cronbach 0,59 que se consideró aceptable por ser una escala multidimensional.

Conclusiones: Se obtuvo un instrumento válido de medida multidimensional en conocimientos, percepción del riesgo y estigmas en tuberculosis en poblaciones privadas de la libertad.

Palabras clave: tuberculosis; conocimientos; percepciones de riesgo; estigma; consenso de experto; prueba piloto.

\begin{abstract}
Background: Tuberculosis is an infectious disease that usually affects the lungs, caused by a bacterium (Mycobacterium tuberculosis). It is transmitted from one person to another through aerosol droplets. This disease is one of the leading causes of mortality worldwide; for this reason, it is relevant to validate an instrument that allows us to evaluate knowledge, risk perceptions and stigmas about tuberculosis.

Objective: To design and validate an instrument to measure knowledge, risk perceptions and stigmas about tuberculosis in the population deprived of liberty.

Methodology: A cross-sectional descriptive observational study was carried out. 11 experts qualitatively and quantitatively evaluated the preliminary instrument. The qualitative evaluation was carried out through a focus group. The quantitative evaluation of the items was carried out using a Likert-type scale, evaluating the following criteria: Coherence, relevance and clarity. And as for the evaluation of the dimensions, this was carried out through the criterion of sufficiency. After the adjustments, the pilot test was carried out on 16 subjects from the penitentiary center.

Results: Of the 20 items initially proposed, the consensus of experts adjusted the structure, eliminating 4 items and adding a new item, leaving the instrument with 17 items. Content validity was evaluated through Aiken's V coefficient $0.9595 \%$ CI (0.820.99); As this was perfect, the reliability was evaluated using Cronbach's Alpha 0.59, which was considered acceptable as it is a multidimensional scale.

Conclusions: A valid multidimensional measurement instrument was obtained in knowledge, risk perception and stigma in tuberculosis in populations deprived of liberty.

Keywords: tuberculosis, knowledge, risk perceptions, stigma, expert consensus, pilot test.

\section{Introducción}

La tuberculosis (TB) continúa siendo un serio problema de salud pública a nivel mundial. Es una enfermedad infectoconta-

giosa de fácil transmisión, siendo una de las 10 principales causas de muerte en todo el mundo y la principal causa de muerte por un agente infeccioso por encima del VIH /SIDA. En el informe mundial de TB
\end{abstract}


para el 2020 se dejó en descubierto la carga de la enfermedad. Para el 2019 estimaron que 10 millones de personas enfermaron de tuberculosis en todo el mundo, con un valor estimado de muertes de 1.4 millones en personas (1). Se estima que más del $87 \%$ de los casos se concentran en países de bajos y medianos ingresos como India, seguido de Indonesia, China, Filipinas, Pakistán, Nigeria y Sudáfrica (2).

En Colombia, según el informe presentado en el 2018 por el Instituto Nacional de Salud (INS), en el Sistema de Vigilancia en Salud Pública (Sivigila) se notificaron 14446 casos de TB de todas las formas; asimismo, la tasa de incidencia de tuberculosis (todas las formas) fue de 26,9 casos por cada 100000 habitantes. Los departamentos con mayor carga fueron Antioquia (18,1\%), Valle del Cauca $(13,3 \%)$ y el distrito de Bogotá $(7,9 \%)$. Estas cifras ubican al departamento y a su capital como la segunda entidad territorial que más aporta tuberculosis en Colombia, lo que connota un mayor riesgo de transmisión en la región (3). Por ello se considera la tuberculosis como una enfermedad reemergente, ya que afecta a población más vulnerable o en riesgo como población privada de la libertad, habitante de calle, población indígena, fuerzas armadas, educadores, trabajadores de la salud y pacientes inmunocomprometidos $(4,5)$. En Santiago de Cali, para el 2018, se notificó al Sistema de Vigilancia 1125 casos de tuberculosis entre nuevos y recaídas, y se obtuvo una incidencia de 46 casos por 100000 habitantes. Dentro de las poblaciones en condiciones de vulnerabilidad y de riesgo con mayor carga de tuberculosis sensible se encontró que, para el año 2019, uno de los grupos con mayor afectación fue la población privada de la libertad con un $12.1 \%$ (183), la población indígena con el 5,2\% (78), habitantes de calle con el 9,8\% (149), migrantes con el $1,9 \%(29)$ y trabajadores de la salud con un 2,8\% (42) (6). Padecer de tuberculosis, para poblaciones en estas condiciones, es definitivamente un factor de empobrecimiento, por la reducción de ingresos familiares (resultado de la disminución del rendimiento para trabajar o estudiar, que repercute en menores ingresos actuales o futuros) y la intensificación de costos catastróficos asumidos por las familias (7).

Para hacer frente a la complejidad de la enfermedad, organizaciones internacionales como la Organización Mundial de la Salud (OMS) y la Organización Panamericana de la Salud (OPS) han instado en adoptar diferentes estrategias en la lucha contra la TB, como los Objetivos del Desarrollo del Milenio (ODM) y con las iniciativas contempladas en el Plan Estratégico Colombia hacia el fin de la TB 2016-2025, donde uno de sus principales objetivos es reducir el $50 \%$ de la incidencia, el $75 \%$ de la mortalidad y el $60 \%$ de los costos catastróficos causados a los afectados y a sus familias por la tuberculosis en Colombia al 2025(8,9). A pesar de los múltiples esfuerzos de la OMS sobre el control de la tuberculosis a nivel mundial, la tuberculosis sigue siendo un reto para el sistema de salud. Múltiples autores han reportado que el éxito está en ampliar el conocimiento y cambiar la percepción de riesgo sobre la tuberculosis. Estos determinantes son especialmente relevantes en poblaciones vulnerables como habitantes de calle y población privada de la liber- 
tad, puesto que una mejora en los conocimientos y en las acciones de autocuidado puede contribuir a mitigar la incidencia de la tuberculosis en estos grupos de riesgo.

En países como Brasil, Etiopía, Bolivia, Ecuador y Paraguay, se han desarrollado y publicado investigaciones sobre conocimiento, percepción del riesgo y estigma de TB en el grupo de riesgo de la población privada de la libertad (PPL) (5). Estos estudios han derivado en el desarrollo de acciones dirigidas a la participación individual y comunitaria, que generan un impacto sobre las prácticas de autocuidado de las personas con TB, al igual que la adherencia y estigma de la enfermedad (10-13). El estigma se conceptualiza como un determinante de desigualdades sociales. Desde esta perspectiva, se evidencia un escenario complejo debido a la confluencia de diversos factores individuales, programáticos, de conocimientos, inmersos en un contexto sociopolítico, económico, educativo, laboral, que determinan el proceso salud-enfermedad, y que encuentra limitantes en el desarrollo de las estrategias del programa de control de TB en su búsqueda, captación, diagnóstico y tratamiento de casos.

El desconocimiento de la enfermedad es uno de los factores que pueden limitar la adherencia, lo cual puede conducir a la generación de mitos, estigmas, temores y estereotipos que se transmiten a los pacientes y sus familias (14).

Según la literatura el conocimiento, la percepción del riesgo y estigma en tuberculosis ha sido estudiado en su mayoría en población no vulnerable o considerada de bajo riesgo para adquirir la enfermedad, y ha sido poco explorado en el gru- po de PPL, un tema ajeno en Colombia y particularmente en la ciudad de Santiago de Cali. Por ello, resulta necesario diseñar y validar un instrumento que mida los conocimientos, percepciones del riesgo y estigma sobre tuberculosis en población privada de la libertad. Con la validación del instrumento se garantiza una herramienta confiable para ser utilizada en otros proyectos que necesiten este tipo de medición.

\section{Materiales y Métodos}

\section{Diseño}

Se realizó un estudio descriptivo transversal para validar un instrumento por medio de consenso de expertos y prueba piloto. El proceso de recolección de la información se realizó entre el periodo de septiembre 2019 - julio 2020.

\section{Participantes}

La población fue conformada en dos escenarios.

Grupo I: Profesionales con experiencia en el tema de estudio con perfiles investigativos y asistenciales. Se convocaron 11 expertos que aceptaron participar voluntariamente en el consenso. Con experiencia en estudios analíticos y trabajo asistencial en TB con poblaciones vulnerables y PPL. En la Tabla 1 se presentan los perfiles de las personas que participaron en el grupo focal.

Grupo II: 16 sujetos en situación de privación de libertad del centro penitenciario Villanueva, Cali Colombia, sin diagnóstico de tuberculosis que aceptaron participar voluntariamente en la realización de la prueba piloto del instrumento a través de consentimiento verbal. La selec- 


\begin{tabular}{|c|c|c|}
\hline $\begin{array}{l}\text { Núme- } \\
\text { ro de } \\
\text { ex- } \\
\text { perto }\end{array}$ & $\begin{array}{l}\text { Formación } \\
\text { académica }\end{array}$ & Experiencia \\
\hline 1 & $\begin{array}{l}\text { Enfermera, epide- } \\
\text { mióloga }\end{array}$ & Experiencia clínica pacientes TB infantil, niños UCI. \\
\hline 2 & Psicóloga & $\begin{array}{l}\text { Área clínica y educativa, asesorías pre y post VIH en pa- } \\
\text { cientes con y sin TB. EPS. Cárcel también en EPS como en } \\
\text { el pregrado. En el pregrado hacía visitas para hacer valora- } \\
\text { ciones en PPL. Área clínica }\end{array}$ \\
\hline 3 & Enfermera & $\begin{array}{l}6 \text { años trabajando en la cárcel, encargada de todos los pro- } \\
\text { gramas incluso TB, contacto en la búsqueda, captación, de- } \\
\text { tección y tratamiento de los pacientes. } 4 \text { años en la Secretaría } \\
\text { de Salud Pública Municipal en el programa de TB y Lepra. } \\
\text { Y lleva } 2 \text { años como referente territorial en PPL }\end{array}$ \\
\hline 4 & Enfermera & $\begin{array}{l}\text { Investigadora del proyecto, experiencia en captación de pa- } \\
\text { cientes con TB }\end{array}$ \\
\hline 5 & Enfermera & Investigadora principal, experiencia en TB área asistencial \\
\hline 6 & Enfermera & Investigadora del proyecto \\
\hline 7 & $\begin{array}{l}\text { Bacterióloga, epi- } \\
\text { demióloga }\end{array}$ & $\begin{array}{l}\text { Apoyo investigativo en TB. Experiencia de estudio de casos } \\
\text { y controles en PPL. }\end{array}$ \\
\hline 8 & Psicóloga & $\begin{array}{l}\text { Experiencia área clínica de tercer nivel. } 3 \text { años en Secretaría } \\
\text { de Salud Pública Municipal en el programa de TB. } 2 \text { años } \\
\text { experiencia en PPL, acercamiento en cárcel, visitas en área } \\
\text { de aislamiento. }\end{array}$ \\
\hline 9 & Enfermera & Estudiante maestría en epidemiología. \\
\hline 10 & $\begin{array}{l}\text { Bacteriólogo, epi- } \\
\text { demiólogo }\end{array}$ & $\begin{array}{l}\text { Investigación en TB. Trabajo con OIM, con Secretarías de } \\
\text { Salud, pertenece en la Alianza TB. Participación a nivel na- } \\
\text { cional con establecimiento de prioridades en investigación en } \\
\text { TB, incluye la concepción del problema, medición e impacto } \\
\text { en PPL. }\end{array}$ \\
\hline 11 & $\begin{array}{l}\text { Antropólogo, so- } \\
\text { ciólogo }\end{array}$ & $\begin{array}{l}4 \text { años investigando en TB. Experiencia en jornadas de salud } \\
\text { con PPL. }\end{array}$ \\
\hline
\end{tabular}

Tabla 1: Fuente: Elaboración propia.

ción de esta muestra fue por conveniencia.

\section{Instrumento}

Se diseñó un instrumento con 20 ítems, agrupados en tres dimensiones: Conoci- miento con nueve ítems, Percepción de riesgo con cuatro ítems y Estigma con siete ítems.

El instrumento fue diseñado por los in- 
vestigadores. La construcción del cuestionario y la redacción de los ítems se realizó con base en la guía $A$ Guide to Developing Knowledge, Attitude and Practice Surveys (15) y experiencia de los investigadores. El instrumento estuvo estructurado con opciones de respuestas abiertas, dicotómicas y de selección múltiple con única o varias respuestas. La evaluación de los ítems agrupados en las tres dimensiones se realizó utilizando el modelo juicio de expertos, propuesta por Escobar Pérez y Cuervo - Martínez (16). La metodología implementada para la calificación de los ítems es de uno a cuatro, donde: 1 . No cumple con el criterio, 2. Bajo nivel, 3. Moderado nivel, 4. Alto nivel, en las categorías de Suficiencia, Claridad, Coherencia y Relevancia, apreciando las observaciones emitidas por los participantes del conceso de experto. (Ver Anexo 1).

\section{Consideraciones Éticas}

Para efectos de la presente investigación, fue evaluado y aprobado por el comité de ética y bioética de investigación de la Universidad Libre, el 2 de abril de 2020.

Para el consenso se contó por escrito con el consentimiento informado de cada uno de los participantes (Ver Anexo 2). Como evidencia se realizó grabación de audio del consenso.

\section{Procedimiento}

Consenso de Expertos El 30 de noviembre de 2019, se convocó a 11 expertos para validar un instrumento que permitiera medir o valorar los conocimientos, las percepciones del riesgo y el nivel de estig- ma sobre la tuberculosis en la población privada de la libertad.

Esta reunión, que tuvo la característica de ser un grupo focal, se realizó en una sala de juntas de la Facultad de Salud de la Universidad Libre, sede Valle del Lili, Cali. El objetivo del grupo focal fue realizar la validación de un instrumento para medir conocimiento, percepción del riesgo y estigma sobre la tuberculosis en población privada de la libertad. De este modo, se buscó obtener una opinión informada de personas con trayectoria en el tema (ya sea en tuberculosis o trabajando con población privada de la libertad u otras poblaciones vulnerables) para adaptar el instrumento al contexto de la población privada de la libertad. En esta medida, se buscó atender a significados culturales propios del contexto carcelario y, de igual manera, reducir la ambigüedad en las interpretaciones de los ítems al momento de ser aplicada la encuesta. El consenso fue realizado por un profesional en el área de antropología y sociología, con experiencia en acompañamiento a grupo focales.

Se pidió a los expertos que evaluaran las preguntas del instrumento desarrollado inicialmente por los investigadores del estudio, teniendo en cuenta que sería aplicado en un contexto de personas con privación de libertad y que debía pensarse en las condiciones en que se encuentra dicha población (su nivel de escolaridad, hacinamiento, sus patrones culturales, sus costumbres dentro de la cárcel). De ese modo, el instrumento podría servir tanto para una población masculina como femenina y también se lograría reducir la ambigüedad al momento de formularla de forma que todos los respondientes apun- 
taran a lo mismo.

La primera parte de la sesión tuvo como objetivo el llegar a un consenso, entre los participantes, sobre la manera de entender los tres constructos que el instrumento busca medir o valorar en el contexto de la población privada de la libertad. Estos son: conocimiento, percepción del riesgo y estigma. A partir de los saberes y experiencias previas de los y las participantes, se buscó establecer una definición operativa para cada constructo según las condiciones y características singulares de la población a la que está dirigida el instrumento. En ese sentido, se parte del supuesto de que medir y/o valorar el conocimiento, la percepción del riesgo y el estigma sobre la tuberculosis debe hacerse de modo diferencial, respecto a cómo se evaluarían estos aspectos en otras poblaciones afectadas por la enfermedad.

La segunda parte de la sesión consistió en una revisión del instrumento original, pregunta a pregunta. Para cada pregunta se recogieron las impresiones del grupo de expertos y expertas en cuanto a sus niveles de claridad, suficiencia y coherencia. El espacio permitió discutir estos criterios para cada pregunta, añadir o remover alguna pregunta y organizar el instrumento para que tuviera una mayor fluidez y orden lógico al momento de preguntar.

Posterior al consenso se realizó los ajustes del instrumento, preservando los juicios cualitativos emitidos por los expertos.

Por comunicación electrónica (correo electrónico) se envió a cada uno de los 11 participantes, el instrumento ajustado con el fin de evaluar cuantitativamente de manera individual la coherencia, relevancia, claridad de cada uno de los ítems y la suficiencia en cada una de las dimensiones; esto utilizando la plantilla "Juicio de expertos" de Escobar -Pérez y Cuervo - Martínez (16). (Ver Anexo 3).

\section{Prueba piloto}

El instrumento obtenido de la validación de contenido por panel de expertos, fue sometido a prueba piloto en una muestra de 16 sujetos en la población de interés, personas privada de la libertad sin diagnóstico de tuberculosis. Se aplicaron dos metodologías en la aplicación de la prueba piloto. La mitad del grupo realizó la encuesta auto diligenciada y en la otra mitad el encuestador realizó las preguntas. Esta estrategia facilitó observar las percepciones de los encuestados con respecto al instrumento, aceptación visual y permitió determinar cuál sería la mejor estrategia de aplicación.

\section{Análisis de información}

Realizada la evaluación por los expertos, se recopila la información de cada uno de ellos y se crea una base de datos en Microsoft Excel 2016, que posteriormente fueron analizados estadísticamente en el mismo programa de Excel, otros estadísticos como el programa SPSS versión 26. Se realizó distribución de frecuencias y media en cada una de las categorías. Se halló el coeficiente $\mathrm{V}$ de AIKEN para la validez del contenido, método que cuantifica la relevancia de cada ítem respecto de un dominio de contenido formulado por $\mathrm{N}$ experto. Su valor oscila de 0 hasta 1 , siendo el valor 1 un perfecto acuerdo entre los expertos respecto a la mayor puntuación de validez de los contenidos evaluados(17). 
La ecuación, algebraicamente modificada por Penfield y Giacobbi (18), es:

$$
V=\frac{\bar{X}-l}{K}
$$

$\bar{X}=$ Es la media de las calificaciones de los expertos en la muestra

$l=$ Es la calificación mas baja posible

$k=$ Es el rango de los valores posibles de la escala Likert utilizada

$K=\mathrm{k}-1$

Para la estimación de los intervalos de confianza del $95 \%$ en los coeficientes V de Aiken se utilizaron las siguientes ecuaciones:

$$
L=\frac{2 n k V+z^{2}-z \sqrt{4 n k V(1-V)+z^{2}}}{2\left(n k+z^{2}\right)}
$$

$$
U=\frac{2 n k V+z^{2}+z \sqrt{4 n k V(1-V)+z^{2}}}{2\left(n k+z^{2}\right)}
$$

Se considera que el coeficiente $\mathrm{V}$ de Aiken es el estadístico pertinente para evaluar la validez del contenido (19), para que el ítem sea considerado válido debe tener un valor V de Aiken superior a 0,70 (20). Un valor por debajo de este, el ítem se elimina.

La consistencia interna del instrumento fue estimada con el método de alfa de Cronbach.

\section{Resultados}

\subsection{Cualitativa}

De acuerdo con el consenso de expertas y expertos, a continuación, se destacan los aspectos relevantes para operativizar cada constructo en un instrumento de investigación en el marco del contexto carcelario:

\section{Conocimiento}

Para el constructo sobre los conocimientos que debería tener la PPL sobre $\mathrm{TB}$, los y las expertas consideraron que el elemento más importante a considerar en el instrumento son los modos de transmisión. En un entorno carcelario hay cierta referencia de lo que es la TB, puesto que hay muchos casos y es común escuchar la palabra en el día a día. Pero, hay muchas percepciones e ideas diversas sobre los modos de transmisión que pueden llevar a hábitos riesgosos o a prácticas estigmatizadoras.

Otro aspecto clave está en la identificación de la sintomatología. En ese sentido, el instrumento debe indagar por el conocimiento que tienen sobre los síntomas de la TB pulmonar (que es más común), más allá de la tos crónica (por más de 15 días). De otro lado, se sugirió que se indagara por el conocimiento de ciertos factores de riesgo (como alcoholismo, desnutrición, consumos de SPA) que pueden hacer que alguien sea más vulnerable a contraer la TB y desarrollar la enfermedad.

Otros asuntos a considerar para operacionalizar el constructo conocimientos sobre la TB en PPL es que el instrumento indague por los siguientes factores: qué es la TB, si conoce el tratamiento y si sabe que tiene cura.

\section{Percepción del riesgo}

Para operativizar este concepto, se parte de conocer el nivel de conciencia de la PPL sobre la propensión a contagiarse de TB. Dado que el riesgo de tener TB es subjetivo (depende de la percepción y de 
las experiencias de la persona), el instrumento debe indagar por cómo perciben la enfermedad quienes no la han tenido para identificar las posibles recomendaciones de comportamientos y hábitos en los que se deba enfatizar para prevenir el contagio. En ese sentido, es poco útil indagar por las condiciones físicas del recinto carcelario que representan un riesgo, dado que no son modificables por los individuos, pero sí es pertinente reconocer qué tan grave consideran a la TB y qué creencias y comportamientos son los que median para pensar en el diseño y desarrollo de posibles acciones dirigidas hacia la prevención y el autocuidado.

\section{Estigma}

Los y las expertas consideraron que las preguntas del instrumento que busquen valorar el estigma deben considerar la distinción entre aquellas acciones encaminadas al control de la TB y aquellas que generan exclusión y discriminación. Es decir, que en la cárcel deben tenerse ciertos cuidados para prevenir la enfermedad, los cuales no deben considerarse prácticas excluyentes. Pero sí debe atenderse a aquellas prácticas que exageran los cuidados y/o rechazan a los enfermos de TB (como llamarlo "tuberculoso", separar todos sus utensilios, aislarlo por completo sin ningún contacto, quemarle las cosas).

Aspectos a considerar para la elaboración y aplicación del instrumento

El nivel de escolaridad es importante para la manera como se formulan las preguntas. Debido a que la mayoría de la población carcelaria cuenta con niveles educativos bajos, las preguntas deben contener un lenguaje claro, sin ambigüedades, que permita a las personas encuestadas comprender lo que se está buscando indagar. Aquí también es clave tener conocimiento de la jerga usada dentro del recinto carcelario. Lo anterior es clave para que, al momento de preguntar, el encuestador pueda utilizar este vocabulario para buscar una mejor comprensión de la pregunta.

Como la población de la cárcel es flotante (todos los días entran y salen personas), se torna clave la capacitación continua sobre TB para alcanzar a la población nueva. Por ello, se aconseja aplicar este instrumento con cierta frecuencia, para analizar qué se debe reforzar a medida que varía la población.

De los 20 ítems en la propuesta inicial, se eliminó 4 ítems (3 de percepción de riesgo y 1 de estigma), de los 16 ítems restantes, 13 de ellos tuvieron ajustes en la redacción y finalmente adicionaron un nuevo ítem en la dimensión de conocimiento para cumplir la totalidad.

El constructo final del instrumento quedó con 17 ítems agrupados en 3 dimensiones teóricas: conocimiento (del ítem 1 al 8), percepción de riesgo (del ítem 9 al 11), estigma (del ítem 12 al 17).

\subsection{Cuantitativa}

Todos los expertos realizaron la evaluación de la escala en su totalidad.

Se calculó el coeficiente $\mathrm{V}$ de Aiken en cada uno de los ítems de acuerdo a las calificaciones emitidas por los profesionales, los ítems del instrumento están enfocados en tres dimensiones; conocimiento, percepción de riesgo y estigma. Cabe resaltar que se utilizó una escala de medición tipo 
Resultados obtenidos por los expertos

\begin{tabular}{|l|l|l|l|l|l|l|l|l|l|l|l|l|l|}
\hline Ítems & E1 & E2 & E3 & E4 & E5 & E6 & E7 & E8 & E9 & E10 & E11 & $\begin{array}{c}\text { V AI- } \\
\text { KEN }\end{array}$ & IC 95 \% \\
\hline $\mathbf{1}$ & 3 & 4 & 4 & 4 & 4 & 4 & 4 & 4 & 4 & 4 & 4 & 0,97 & $0,85-0,99$ \\
\hline $\mathbf{2}$ & 4 & 4 & 4 & 4 & 4 & 4 & 4 & 4 & 4 & 4 & 4 & 1 & $0,90-1,00$ \\
\hline $\mathbf{3}$ & 4 & 4 & 4 & 4 & 4 & 4 & 4 & 4 & 4 & 4 & 4 & 1 & $0,90-1,00$ \\
\hline $\mathbf{4}$ & 4 & 4 & 4 & 4 & 4 & 4 & 4 & 4 & 4 & 4 & 4 & 1 & $0,90-1,00$ \\
\hline $\mathbf{5}$ & 2 & 4 & 4 & 4 & 4 & 4 & 4 & 4 & 3 & 4 & 4 & 0,89 & $0,74-0,96$ \\
\hline $\mathbf{6}$ & 4 & 4 & 4 & 4 & 4 & 4 & 4 & 4 & 3 & 4 & 4 & 0,97 & $0,85-0,99$ \\
\hline $\mathbf{7}$ & 4 & 4 & 4 & 4 & 4 & 4 & 4 & 4 & 3 & 4 & 3 & 0,93 & $0,79-0,98$ \\
\hline $\mathbf{8}$ & 4 & 4 & 4 & 4 & 4 & 4 & 4 & 4 & 4 & 4 & 4 & 1 & $0,90-1,00$ \\
\hline $\mathbf{9}$ & 4 & 4 & 4 & 4 & 4 & 4 & 4 & 4 & 4 & 4 & 4 & 1 & $0,90-1,00$ \\
\hline $\mathbf{1 0}$ & 4 & 4 & 4 & 4 & 4 & 4 & 4 & 4 & 3 & 4 & 4 & 0,97 & $0,85-0,99$ \\
\hline $\mathbf{1 1}$ & 4 & 4 & 4 & 4 & 4 & 4 & 4 & 4 & 4 & 4 & 4 & 1 & $0,90-1,00$ \\
\hline $\mathbf{1 2}$ & 2 & 4 & 4 & 4 & 4 & 4 & 4 & 4 & 4 & 4 & 4 & 0,92 & $0,78-0,97$ \\
\hline $\mathbf{1 3}$ & 4 & 4 & 4 & 4 & 3 & 4 & 4 & 4 & 4 & 4 & 4 & 0,97 & $0,85-0,99$ \\
\hline $\mathbf{1 4}$ & 4 & 4 & 4 & 4 & 4 & 4 & 4 & 4 & 4 & 4 & 4 & 1 & $0,90-1,00$ \\
\hline $\mathbf{1 5}$ & 4 & 4 & 4 & 4 & 3 & 4 & 4 & 4 & 4 & 4 & 4 & 0,97 & $0,85-0,99$ \\
\hline $\mathbf{1 6}$ & 4 & 4 & 4 & 4 & 4 & 4 & 4 & 4 & 4 & 4 & 4 & 1 & $0,90-1,00$ \\
\hline $\mathbf{1 7}$ & 4 & 4 & 4 & 4 & 4 & 4 & 4 & 4 & 4 & 4 & 3 & 0,97 & $0,85-0,99$ \\
\hline Total & & & & & & & & & & & & 0,98 & $0,86-1,00$ \\
\hline
\end{tabular}

Tabla 2: Resultados de la validez de contenido en la coherencia.

Likert con cuatro categorías.

En la Tabla 2 Se observa que el coeficiente $\mathrm{V}$ de Aiken en la categoría de coherencia los 17 ítems presentaron validez de contenido, los valores son superiores a 0,80 y El indicador de coherencia global tiene un coeficiente de Aiken de 0,98 IC 95\% $(0,86-1,00)$. En esta categoría los 11 expertos evaluaron 8 ítems con alto nivel.

En los resultados de la validez de contenido en la relevancia (Ver Tabla 3), observamos que la mayoría de los ítems tienen un coeficiente $\mathrm{V}$ de Aiken superior de 0,80, excepto el ítem 10 que tiene un $\mathrm{V}$ de Aiken en 0,79 . No se eliminó este ítem, pero se realizó ajuste en la redacción según las observaciones cualitativas emitidas por los expertos. El indicador de relevancia global tiene un coeficiente $\mathrm{V}$ de Aiken de $0,92 \mathrm{IC}$ $95 \%(0,78-0,97)$.

En la Tabla 4 de resultados de la validez de contenido en la claridad, apreciamos que los 17 ítems cumplen con la validez del contenido de la escala, todos tienes coeficientes $\mathrm{V}$ de Aiken también superiores de 0,80. El global para este indicador de claridad en V de Aiken 0,96 IC 95\% $(0,83-0,99)$.

Posteriormente, se evaluaron las observaciones cualitativas de los expertos con- 
Resultados obtenidos por los expertos

\begin{tabular}{|l|l|l|l|l|l|l|l|l|l|l|l|l|l|}
\hline Ítems & E1 & E2 & E3 & E4 & E5 & E6 & E7 & E8 & E9 & E10 & E11 & $\begin{array}{c}\text { V AI- } \\
\text { KEN }\end{array}$ & IC 95 \% \\
\hline $\mathbf{1}$ & 4 & 4 & 4 & 4 & 4 & 4 & 4 & 4 & 4 & 4 & 4 & 1 & $0,90-1,00$ \\
\hline $\mathbf{2}$ & 4 & 3 & 4 & 4 & 4 & 3 & 4 & 4 & 4 & 4 & 4 & 0,93 & $0,79-0,98$ \\
\hline $\mathbf{3}$ & 4 & 4 & 4 & 4 & 4 & 4 & 4 & 4 & 4 & 4 & 4 & 1 & $0,90-1,00$ \\
\hline $\mathbf{4}$ & 4 & 4 & 4 & 4 & 4 & 4 & 4 & 4 & 4 & 4 & 4 & 1 & $0,90-1,00$ \\
\hline $\mathbf{5}$ & 2 & 4 & 4 & 4 & 4 & 4 & 4 & 4 & 4 & 4 & 4 & 0,92 & $0,78-0,97$ \\
\hline $\mathbf{6}$ & 4 & 4 & 4 & 4 & 4 & 4 & 4 & 4 & 4 & 4 & 4 & 1 & $0,90-1,00$ \\
\hline $\mathbf{7}$ & 4 & 4 & 3 & 4 & 4 & 4 & 4 & 4 & 4 & 4 & 4 & 0,97 & $0,85-0,99$ \\
\hline $\mathbf{8}$ & 4 & 4 & 4 & 4 & 4 & 4 & 4 & 4 & 3 & 4 & 4 & 0,97 & $0,85-0,99$ \\
\hline $\mathbf{9}$ & 4 & 4 & 4 & 4 & 2 & 4 & 4 & 4 & 3 & 4 & 4 & 0,89 & $0,74-0,96$ \\
\hline $\mathbf{1 0}$ & 3 & 4 & 3 & 4 & 2 & 3 & 4 & 4 & 3 & 4 & 4 & 0,79 & $0,62-0,89$ \\
\hline $\mathbf{1 1}$ & 4 & 3 & 4 & 4 & 4 & 4 & 4 & 4 & 4 & 4 & 4 & 0,97 & $0,85-0,99$ \\
\hline $\mathbf{1 2}$ & 2 & 4 & 4 & 4 & 4 & 3 & 4 & 4 & 3 & 4 & 4 & 0,85 & $0,69-0,93$ \\
\hline $\mathbf{1 3}$ & 4 & 4 & 4 & 4 & 3 & 4 & 4 & 4 & 3 & 4 & 4 & 0,93 & $0,79-0,98$ \\
\hline $\mathbf{1 4}$ & 4 & 2 & 4 & 4 & 4 & 4 & 4 & 4 & 3 & 4 & 4 & 0,89 & $0,74-0,96$ \\
\hline $\mathbf{1 5}$ & 3 & 4 & 4 & 4 & 3 & 4 & 4 & 4 & 3 & 4 & 4 & 0,90 & $0,75-0,96$ \\
\hline $\mathbf{1 6}$ & 4 & 4 & 4 & 4 & 4 & 3 & 4 & 4 & 3 & 4 & 4 & 0,93 & $0,79-0,98$ \\
\hline $\mathbf{1 7}$ & 4 & 4 & 4 & 4 & 4 & 4 & 4 & 4 & 3 & 4 & 3 & 0,93 & $0,79-0,98$ \\
\hline Total & & & & & & & & & & & & 0,92 & $0,78-0,97$ \\
\hline
\end{tabular}

Tabla 3: Resultados de la validez de contenido en la relevancia.

signadas en la herramienta evaluativa enviada por correo electrónico. Estas se analizaron de manera global y se tomaron en cuenta los ítems que mayores apreciaciones tenían.

El ítem 10 que obtuvo un coeficiente $\mathrm{V}$ de Aiken de 0,79 en relevancia se ajustó en su redacción donde se modifica la palabra prevenir por evitar (ver instrumentos en anexos).

Cada ítem fue evaluado en la coherencia, relevancia y claridad. La evaluación de las dimensiones de la escala se realizó por medio de la categoría de suficiencia que nos permitió evaluar que los ítems que pertenecen a una misma dimensión bastan para obtener la medición de esta; se utilizó la misma escala de medición. (Ver Tabla 5).

Observamos que los ítems que conforman cada dimensión tienen validez del contenido y valoran lo que realmente quieren valorar. El coeficiente $\mathrm{V}$ de Aiken en las dimensiones son superiores a 0,80 . Con los resultados reportados anteriormente, estructuración de algunos ítems y las observaciones referidas por los expertos permitieron obtener un instrumento más claro.

Se realizó el análisis de confiabilidad del 


\section{Resultados obtenidos por los expertos}

\begin{tabular}{|l|l|l|l|l|l|l|l|l|l|l|l|l|l|}
\hline Ítems & E1 & E2 & E3 & E4 & E5 & E6 & E7 & E8 & E9 & E10 & E11 & $\begin{array}{c}\text { V AI- } \\
\text { KEN }\end{array}$ & IC 95 \% \\
\hline $\mathbf{1}$ & 4 & 4 & 4 & 4 & 4 & 4 & 4 & 4 & 4 & 4 & 4 & 1 & $0,90-1,00$ \\
\hline $\mathbf{2}$ & 4 & 4 & 3 & 3 & 4 & 4 & 4 & 4 & 4 & 3 & 4 & 0,90 & $0,75-0,96$ \\
\hline $\mathbf{3}$ & 4 & 4 & 4 & 4 & 4 & 4 & 4 & 4 & 4 & 3 & 4 & 0,97 & $0,85-0,99$ \\
\hline $\mathbf{4}$ & 4 & 4 & 4 & 3 & 4 & 4 & 4 & 4 & 4 & 4 & 4 & 0,97 & $0,85-0,99$ \\
\hline $\mathbf{5}$ & 2 & 4 & 4 & 4 & 4 & 4 & 4 & 4 & 4 & 4 & 4 & 0,92 & $0,78-0,97$ \\
\hline $\mathbf{6}$ & 4 & 4 & 4 & 4 & 4 & 4 & 4 & 4 & 4 & 4 & 4 & 1 & $0,90-1,00$ \\
\hline $\mathbf{7}$ & 4 & 4 & 4 & 4 & 4 & 4 & 4 & 4 & 4 & 3 & 4 & 0,97 & $0,85-0,99$ \\
\hline $\mathbf{8}$ & 4 & 4 & 4 & 4 & 4 & 4 & 4 & 4 & 4 & 4 & 4 & 1 & $0,90-1,00$ \\
\hline $\mathbf{9}$ & 4 & 4 & 4 & 4 & 4 & 4 & 4 & 4 & 4 & 4 & 4 & 1 & $0,90-1,00$ \\
\hline $\mathbf{1 0}$ & 3 & 4 & 4 & 4 & 4 & 4 & 4 & 4 & 4 & 3 & 4 & 0,93 & $0,79-0,98$ \\
\hline $\mathbf{1 1}$ & 4 & 4 & 4 & 4 & 4 & 4 & 4 & 4 & 4 & 4 & 4 & 1 & $0,90-1,00$ \\
\hline $\mathbf{1 2}$ & 2 & 4 & 3 & 4 & 4 & 4 & 4 & 4 & 3 & 4 & 4 & 0,85 & $0,69-0,93$ \\
\hline $\mathbf{1 3}$ & 4 & 4 & 4 & 4 & 4 & 4 & 4 & 4 & 4 & 4 & 3 & 0,97 & $0,85-0,99$ \\
\hline $\mathbf{1 4}$ & 4 & 4 & 4 & 4 & 4 & 4 & 4 & 4 & 4 & 4 & 4 & 1 & $0,90-1,00$ \\
\hline $\mathbf{1 5}$ & 3 & 4 & 4 & 4 & 3 & 4 & 4 & 4 & 4 & 4 & 4 & 0,93 & $0,79-0,98$ \\
\hline $\mathbf{1 6}$ & 4 & 4 & 4 & 3 & 4 & 4 & 3 & 4 & 4 & 3 & 4 & 0,90 & $0,75-0,96$ \\
\hline $\mathbf{1 7}$ & 4 & 4 & 4 & 4 & 4 & 4 & 3 & 4 & 4 & 3 & 3 & 0,90 & $0,75-0,96$ \\
\hline Total & & & & & & & & & & & & 0,96 & $0,83-0,99$ \\
\hline
\end{tabular}

Tabla 4: Resultados de la validez de contenido en la claridad.

Fuente: Elaboración propia a partir de los resultados obtenidos de la evaluación cuantitativa de los expertos.

\section{Resultados obtenidos por los expertos}

\begin{tabular}{|l|l|l|l|l|l|l|l|l|l|l|l|l|l|}
\hline Dimensión & E1 & E2 & E3 & E4 & E5 & E6 & E7 & E8 & E9 & E10 & E11 & $\begin{array}{c}\text { V Ai- } \\
\text { ken }\end{array}$ & IC 95 \% \\
\hline Conocimiento & 4 & 4 & 4 & 4 & 4 & 3 & 4 & 4 & 4 & 4 & 4 & 0,97 & $0,85-0,99$ \\
\hline $\begin{array}{l}\text { Percepción } \\
\text { del Riesgo }\end{array}$ & 4 & 4 & 3 & 4 & 4 & 3 & 4 & 4 & 4 & 4 & 3 & 0,90 & $0,75-0,96$ \\
\hline Estigma & 4 & 4 & 4 & 4 & 4 & 4 & 4 & 4 & 4 & 4 & 4 & 1,00 & $0,90-1,00$ \\
\hline Total & & & & & & & & & & & & 0,95 & $0,82-0,99$ \\
\hline
\end{tabular}

Tabla 5: Resultados de la validez de contenido en la suficiencia.

instrumento mediante el coeficiente Alfa de Cronbach. Consideramos que la fiabili- dad de la escala es moderada (Ver Tabla $6)$, teniendo en cuenta que se contó con 


\section{Estadísticas de fiabilidad}

\begin{tabular}{|l|l|}
\hline Alfa de Cronbach & N de elementos \\
\hline 0,590 & 17 \\
\hline
\end{tabular}

Tabla 6: Alfa de Cronbach de la escala.

solo 17 ítems y una escala multidimensional.

Según Ruiz, una manera de saber hasta dónde el coeficiente de confiabilidad moderado puede ser aceptable, es comparar la desviación estándar de la distribución de puntajes con el error estándar de medición y que se cumpla con la condición de que la desviación estándar de la distribución sea mayor al error estándar de la medición (20). En este caso, el error estándar es de 1,560 y la desviación estándar 5,173, lo que cumple con el criterio. (Ver Tabla 7).

Con la versión final del instrumento validado, se aplicó la prueba piloto en un centro penitenciario de Cali con 16 sujetos, en la que el entrevistador aplica el instrumento directamente a 8 sujetos y al resto hace entrega del instrumento para que sean autodiligenciadas. El encuestador, previamente capacitado, con experiencia en el campo de tuberculosis y trabajos en poblaciones privada de la libertad, emitió una evaluación cualitativa en cuanto el proceso de la prueba piloto. $\mathrm{Al}$ referirse que con ambas metodologías utilizadas hubo aceptación en la escala, comprensión en los ítems, pero la manera útil de aplicarla en cuanto tiempo de realización es por medio de la entrevista (realizada por el encuestador), el tiempo empleado fue de aproximadamente $6 \mathrm{minu}-$ tos. En comparación con las auto diligen- ciadas, que tuvieron un tiempo de $10 \mathrm{mi}-$ nutos, aproximadamente.

La prueba piloto permitió ajustar algunos ítems y apariencia del instrumento. Los ítems 5, 6 y 7 fueron ajustados, donde las opciones contagiar, pegar o prender, fueron unificadas en un solo término, contagiar.

También surgieron los ajustes de apariencia para una mejor aceptación visual del instrumento, como reducir espacios en interlineados, impresiones a doble cara y numeración de las hojas.

\section{Discusión}

El presente trabajo tuvo como objetivo diseñar y validar un instrumento de medición que evalúe conocimiento, percepciones de riesgo y estigma sobre tuberculosis en la población privada de la libertad, población que está más vulnerable de contraer esta enfermedad por el entorno en que se encuentra.

La validación del instrumento se obtuvo por medio del consenso de expertos que tuvo una evaluación cualitativa y cuantitativa. Gracias a estas evaluaciones se obtuvo un instrumento con 17 ítems.

El análisis cuantitativo se determinó por medio del coeficiente $\mathrm{V}$ de Aiken con sus respectivos intervalos de confianza y Alfa de Cronbach. El instrumento tuvo 


\section{Estadísticos descriptivos}

\begin{tabular}{|l|l|l|l|l|l|l|}
\hline & N & Mínimo & Máximo & Media & \multicolumn{2}{|l|}{ Desviación } \\
\hline & Estadístico & Estadístico & Estadístico & Estadístico & Desv. Error & Estadístico \\
\hline Total & 11 & 187 & 204 & 197,82 & 1,56 & 5,173 \\
\hline $\begin{array}{l}\text { N válido } \\
\text { (por lista) }\end{array}$ & 11 & & & & & \\
\hline
\end{tabular}

Tabla 7: Desviación estándar y Error estándar de la medición.

un $\mathrm{V}$ de Aiken de 0,95, demostrando validez del contenido y una fiabilidad de 0,59 y considerándose aceptable para este tipo de estudio.

En la prueba piloto se observó una buena comprensión de los ítems por parte de los participantes, que aprueba la validez de apariencia del instrumento. La mejor manera de su aplicación es por medio de la entrevista.

En relación a validación y confiabilidad de un instrumento se encontró algunos estudios que utilizaron panel de expertos, coeficiente de V de Aiken y Alfa de Cronbach.

En el estudio "Validez y confiabilidad" del cuestionario Prácticas de cuidado que realizan consigo mismas las mujeres en el posparto, donde participaron 10 expertas, quienes evaluaron cada uno de los ítems teniendo en cuenta los criterios de pertinencia y relevancia. En este, el cuestionario presento un índice de validez del contenido 0,88 y confiabilidad por el Alfa de Cronbach de 0,59 en el cuestionario de 32 ítems y en el cuestionario de 12 ítems un Alfa de Cronbach de 0,68, considerándose puntajes aceptable y bueno (21).

Hubo otro estudio titulado "Diseño y validación", de un cuestionario de satisfacción con programas deportivos en cen- tros penitenciarios. El cuestionario (63 ítems) fue analizado cuantitativa y cualitativamente por un grupo de 11 jueces expertos. La validez de contenido se evaluó a través del coeficiente $\mathrm{V}$ de Aiken, y obtuvo resultados por encima del 0,70, consistencia interna por el método del a de Cronbach (0.928) y la fiabilidad testretest utilizando el coeficiente correlación intraclase $0.957(\mathrm{p}<0.01)(22)$.

Instrumentos de medición: conocimientos, actitudes y prácticas en personas con tuberculosis pulmonar, es un estudio donde los investigadores se basaron en 4 fases.

Para el proceso de construcción y validación del instrumento: dos de ellas que también fueron utilizadas en este estudio fueron la validez de contenido y confiabilidad (23).

Una de las debilidades de este estudio hace referencia al número de ítems del instrumento. Mientras mayor sea el número de ítems, el valor del coeficiente incrementara (24). Se resalta que entre los expertos y expertas se decidió que el instrumento debía ser corto para que la aplicación no se demorara debido a las dificultades que se pueden presentar en el entorno carcelario. Otra limitación es que no se realizo una prueba test retest.

Ser una investigación independiente, 
multidisciplinaria fue nuestra fortaleza. Se entregó un instrumento confiable que ya fue aplicado por otro grupo de investigadores donde realizan análisis cuantitativo.

\section{Conclusiones}

Esta fue la primera investigación en Cali en validar un instrumento que mida tres dimensiones (conocimientos, percepciones de riesgos y estigma sobre tuberculosis en poblaciones privada de la libertad).

El aplicar este instrumento permitirá tener otra visión en las acciones de control en los programas de tuberculosis en esta población.

\section{Agradecimientos}

Los autores agradecemos a cada uno de los expertos que hicieron parte del consenso, a la secretaria de salud pública municipal e INPEC por permitir la realización de la prueba piloto en la población privada de la libertad del Centro Penitenciario Villa Hermosa de la ciudad de Cali.

Conflictos de interés: Los autores declaran no tener conflicto de interés.

\section{Referencias}

(1) World Health Organization. WHO Report Global Tuberculosis Control 2019, disponible:https://www. who.int/teams/global-tuberculosisprogramme/tb-reports/globaltuberculosis-report-2020.

(2) Organización Mundial de la Salud. Informe mundial sobre la tu- berculosis 2019 - Sinopsis. Disponible: https://www.who.int/tb/ publications/global_report/es/.

(3) Instituto Nacional de Salud. Informe de evento de tuberculosis. Colombia, 2018, Disponible en: https://www. ins.gov.co/buscadoreventos/ Informesdeevento/ TUBERCULOSIS_2018.

(4) Castañeda-Hernández, D. M., Tobón-García, D., y RodríguezMorales, A. J. (2013). "Asociación entre incidencia de tuberculosis e índice de desarrollo humano en 165 países del mundo." Revista Peruana de Medicina Experimental y Salud Pública, 30, 560-568.

(5) Martínez, M. L. O., Duran, M. E. M., García, O. E. P., Bonilla, H. Q., y Pérez, S. F. "Protocolo de Vigilancia en Salud Pública". Tuberculosis Farmacorresistente.

(6) Secretaria de Salud Municipal De Santiago de Cali. Instituto Nacional de Salud. Informe de evento de tuberculosis. Santiago de Cali, Colombia, 2018

(7) Dirección General de Salud de las Personas Ministerio de Salud LimaPerú 2012. Impacto socioeconómico de la tuberculosis en el Perú 2010.

(8) Ministerios de salud. Plan Estratégico Colombia. Hacia el Fin de la Tuberculosis, 2016-2025.Disponible: https://www.minsalud.gov.co/ 
sites/rid/Lists/BibliotecaDigital/

RIDE/INEC/INTOR/Plan-

estrategico-fin-tuberculosis-

colombia-2016-2025.pdf

(9) Objetivos de Desarrollo del Milenio (ODM).

(10) Ferreira Júnior S, Oliveira HB de, Marin-Léon L, Ferreira Júnior S, Oliveira HB de,Marin-Léon L. "Conhecimento, atitudes e práticas sobre tuberculose em prisões e no serviço público de saúde". Rev Bras Epidemiol. 2013 Mar;16(1):100-13. http://dx.doi.org/10.1590/S1415790X2013000100010

(11) Santos MN de A, Sá AMM, Santos MN de A, Sá AMM. "The carrierbeing of tuberculosis in prisons: a nursing study". Esc Anna Nery - Rev Enferm. 2014;18(2):350-5. http://dx.doi.org/10.5935/14148145.20140051 \protect \unhbox \ voidb@x \penalty $\ @ M \backslash$

(12) Adane K, Spigt M, Johanna L, Noortje D, Abera SF, Dinant G-J. "Tuberculosis knowledge, attitudes, and practices among northern Ethiopian prisoners: Implications for TB control efforts." PLoS One. 2017;12(3):e0174692. https://doi. org/10.1371/journal.pone.0174692

(13) Waisbord S. "Participatory communication for tuberculosis control in prisons in Bolivia, Ecuador, and Paraguay." Rev Panam Salud Pública. 2010 Mar;27(3):168-74.

(14) Universidad Nacional de Colombia. Facultad de Enfermería.
ÓC, Martínez ÓAC, Suancha ÉLF, Sánchez AIM. "Avances en enfermería." Vol. 29, Avances en Enfermería. Facultad de Enfermería, Universidad Nacional de Colombia; 2011. 143-151 p. DOI: $10.15446 /$ av.enferm

(15) World Health Organization. Advocacy, communication and social mobilization for TB control: a guide to developing knowledge, attitude and practice surveys (No. WHO/HTM/STB/2008.46).

(16) Escobar-p J. Validez de contenido y juicio de expertos: Una aproximación a su utilización. 2017; (January 2008). https://doi.org/10. 32870/ap.v9n2.993.

(17) Aiken, L. R. (1985). "Three coefficients for analyzing the reliability and validity of ratings." Educational and psychological measurement, 45(1), 131-142. https://doi. org/10.1177/0013164485451012.

(18) Penfield RD, Miller JM. Applied Measurement in Education Improving Content Validation Studies Using an Asymmetric Confidence Interval for the Mean of Expert Ratings. 2010;(December2014):3741. https://doi.org/10.1207/ s15324818ame1704_2

(19) Binomial P. Criterio de Jueces. Luis Miguel Escurra M.". :103-11.

(20) Merino Soto C, Livia Segovia J. "Intervalos de confianza asimétricos 
para el índice de la validez de contenido: un programa Visual Basic para la V de Aiken." An. psicol. 1 de enero 2009;25(1):169-71. Disponible en: https://revistas.um.es/ analesps/article/view/71631

(21) Vargas Porras C, Hernández Molina LM. «Validez y confiabilidad del cuestionario "Prácticas de cuidado que realizan consigo mismas las mujeres en el posparto"». Av. enferm. [Internet]. 1 de enero de 2010;28(1):96-106. Disponible: https://revistas.unal.edu.co/index. php/avenferm/article/view/15659

(22) Fuentes-guerra FJG, Javier L, González D, Martín JF, Concepción ANA, Sánchez J, et al. Design and Validation of a Satisfaction Questionnaire with Sports Programmes in Penitentiaries (Diseño y validación de un cuestionario de satisfacción con programas deportivos en centros penitenciarios). 2018;21-33.

(23) Muñoz-Sánchez AI, RubianoMesa YL, Saavedra-Cantor CJ. "Measuring instrument: knowledge, attitudes and practices of people with pulmonary tuberculosis." Rev. Latino-Am. Enfermagem.2019;27:e3086. DOI: http://dx.doi.org/10.1590/15188345.2608.3086

(24) Cervantes H. Interpretaciones del coeficiente alpha de Cronbach. $2005 ; 9-28$ 


\section{ANEXO 1: PLANTILLA JUICIO DE EXPERTO}

NOMBRE Y APELLIDO DEL JUEZ:

C.C:

OCUPACIÓN:

\begin{tabular}{|c|c|c|}
\hline SUFICIENCIA & 1 No cumple con el criterid & Los ítems no son suficientes para medir la dimensión \\
\hline \multirow{3}{*}{$\begin{array}{l}\text { Los ítems que pertenecen } \\
\text { a una misma dimensión } \\
\text { bastan para obtener la } \\
\text { medición de ésta }\end{array}$} & 2. Bajo Nivel & $\begin{array}{l}\text { Los ítems mi den algún aspecto de la dimensión pero no corresponden con la } \\
\text { dimensión total }\end{array}$ \\
\hline & 3. Moderado nivel & $\begin{array}{l}\text { Se deben incrementar algunos ítems para poder evaluar la dimensión } \\
\text { completamente. }\end{array}$ \\
\hline & 4. Alto nivel & Los ítems son suftientes \\
\hline CLARIDAD & \multicolumn{2}{|c|}{1 No cumple con el criteridEl ítem no es claro } \\
\hline \multirow{3}{*}{$\begin{array}{l}\text { El ítem se comprende } \\
\text { fácilmente, es decir, su } \\
\text { sintáctica y semántica son } \\
\text { adecuadas. }\end{array}$} & 2. Bajo Nivel & $\begin{array}{l}\text { El ítem requiere bastantes modifíaciones o una modifíación muy grande en } \\
\text { el uso de las palabras de acuerdo con su si gni fíad o o por la ordenación de las } \\
\text { mismas. }\end{array}$ \\
\hline & 3. Moderado nivel & $\begin{array}{l}\text { Se requiere una modificación muy específica de al gunos de los términos del } \\
\text { item }\end{array}$ \\
\hline & 4. Alto nivel & El ítem es claro, téne semántća y sintaxis adecuada. \\
\hline
\end{tabular}

\begin{tabular}{|l|l|l|}
\hline \multicolumn{1}{|c|}{ COHERENCIA } & 1 No cumple con el criteridEl ítem no téne relación lógica con la di mensión \\
\hline $\begin{array}{l}\text { El ítem téne relación } \\
\text { lógica con la dimensión o } \\
\text { indicador que está } \\
\text { midiendo. }\end{array}$ & 2. Bajo Nivel & El ítem téne una relación tangencial con la dimensión. \\
\cline { 2 - 3 } & 3. Moderado nivel & El ítem téne una relación moderada con la dimensión que está mi diendo. \\
\cline { 2 - 3 } & 4. Alto nivel & $\begin{array}{l}\text { El ítem se encuentra completamente relacionado con la di mensión que está } \\
\text { midiendo }\end{array}$ \\
\hline
\end{tabular}

\begin{tabular}{|l|l|l|}
\hline \multicolumn{1}{|c|}{ RELEVANCIA } & 1 No cumple con el criteri & $\begin{array}{l}\text { El ítem puede ser eli mi nado sin que se vea afectada la medición de la } \\
\text { di mensión }\end{array}$ \\
\hline \multirow{2}{*}{$\begin{array}{l}\text { El ítem es esencial o } \\
\text { importante, es decir de be } \\
\text { ser incluido. }\end{array}$} & 2. Bajo Nivel & $\begin{array}{l}\text { El ítem téne al guna relevancia, pero otro ítem puede estar incluyendo lo que } \\
\text { mide éste. }\end{array}$ \\
\cline { 2 - 4 } & \begin{tabular}{l} 
3. Moderado nivel \\
\cline { 2 - 3 }
\end{tabular} & El ítem es relativamente importante. \\
\hline
\end{tabular}


Ahumada et al.

\title{
ANEXO 2: CONSENTIMIENTO INFORMADO \\ PARA PARTICIPACIÓN EN PROYECTO DE INVESTIGACIÓN CONSENSO DE EXPERTOS
}

\author{
Propósito del estudio \\ El proyecto Validación de un instrumento para medir conocimientos, percepción de riesgo \\ y estigmas sobre tuberculosis, tiene como objetivo diseñar y validar un instrumento. Lo \\ anterior con el propósito de garantizar una herramienta confiable para ser utilizada en \\ otros proyectos que necesiten este tipo de medición
}

Desarrollo del estudio

Usted ha sido invitado al consenso de expertos Validación de un instrumento para medir conocimientos, percepción de riesgo y estigmas sobre tuberculosis, el cual consistirá en un grupo focal de discusión para validar un instrumento. Si usted acepta participar en el mismo, en el taller se harán una serie de ejercicios de investigación cualitativa orientados a indagar sobre sus percepciones, opiniones y experiencia sobre conocimientos, percepción de riesgo y estigma sobre tuberculosis para llegar a un consenso con los demás expertos, con el fin de ajustar y validar un instrumento que tiene como objeto ser implementada en una investigación donde la población de estudio son personas privada de la libertad. El taller se realizará entre las 8:00 a. m. y las 11:00 a. m. e incluye espacios de receso.

\section{Confidencialidad y uso de la información}

Toda la información que usted aporte durante la realización del taller será utilizada únicamente con fines de investigación. Habrá espacios en los que se grabarán las discusiones y/o presentaciones que se desarrollen, los cuales serán anunciados con anticipación durante el taller. Toda la información que se recoja durante el tiempo que dure el taller no podrá ser vinculada con usted ni con la institución. Los resultados de este estudio podrán ser presentados en congresos, ponencias o publicaciones científicas, pero su identidad permanecerá protegida y su privacidad será preservada. Solamente el equipo investigador tendrá acceso a la información recogida y a las grabaciones, garantizando que tanto su nombre como sus datos personales no aparecerán en ningún reporte o publicación. Por ello, hemos tomado todas las medidas necesarias para mantener en estricta confidencialidad a todos los participantes de esta investigación.

\section{Participación voluntaria}

Su participación en el estudio es absolutamente voluntaria; puede negarse a participar, a contestar alguna pregunta en particular o retirarse de la investigación en cualquier momento. Usted recibirá una copia de este documento.

Con su firma usted acepta que ha decidido participar en este estudio después de haber

leído este documento, que se han respondido satisfactoriamente a sus preguntas, aclarado sus posibles dudas y que su participación es totalmente voluntaria.

\section{CONSENTIMIENTO INFORMADO}

Doy mi consentimiento para que se usen los datos que comunicaré durante la realización del 
consenso "Validación de un instrumento para medir conocimientos, percepción de riesgo y estigmas sobre tuberculosis". Los resultados de esta investigación son confidenciales. Cualquier pregunta que me surja durante mi participación podrá y deberá ser aclarada por los encargados del estudio.

Acepto participar en esta investigación y mi nombre y firma son:

Nombre Participante

Nombre Representante del equipo de investigación

Nombre Testigo

Usted recibirá una copia del presente documento.

\section{Firma}

Firma

Firma 


\section{ANEXO 3: FORMATO PARA LA EVALUACIÓN CUAN- TITATIVA DE LOS ÍTEMS.}

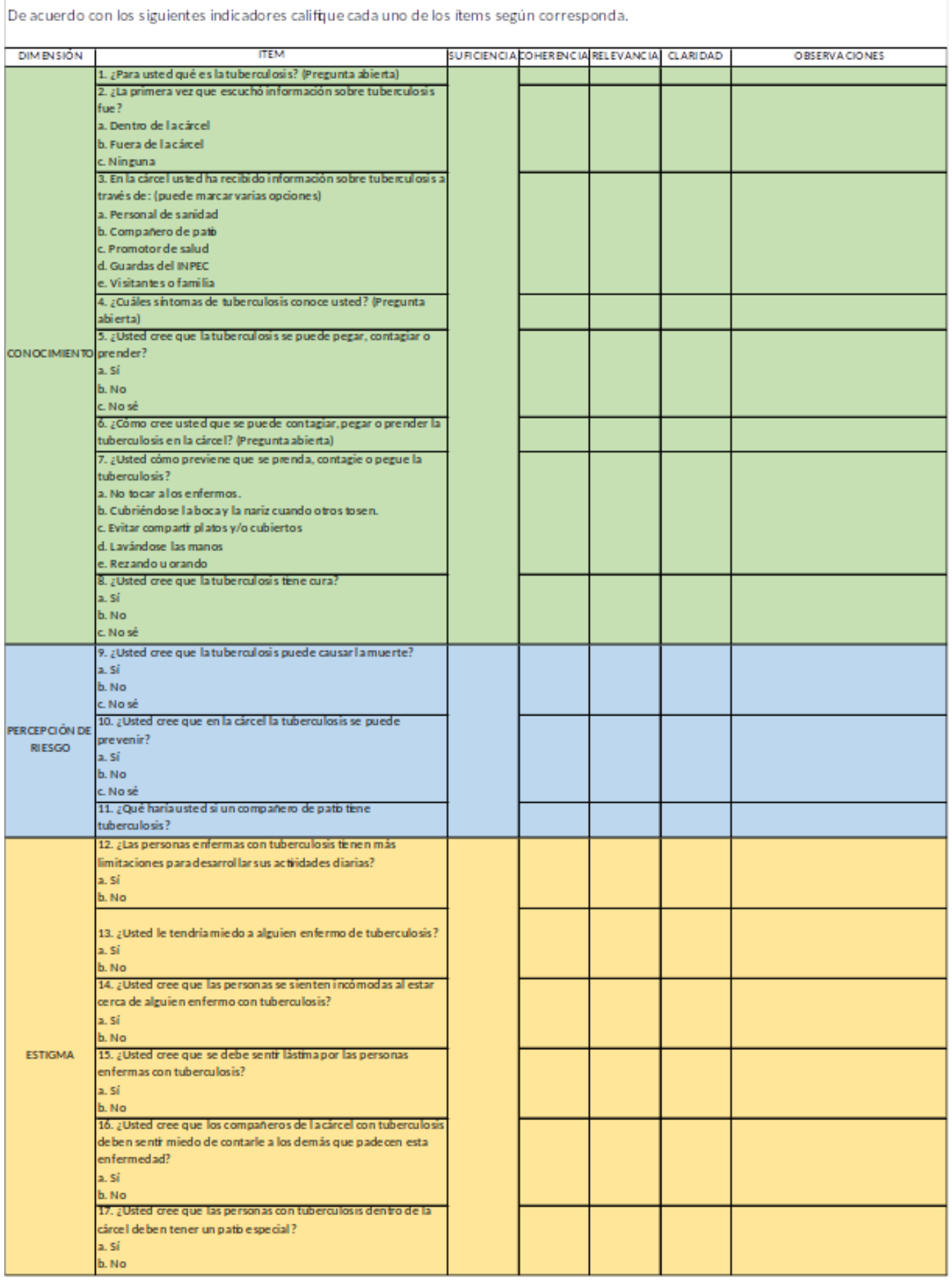




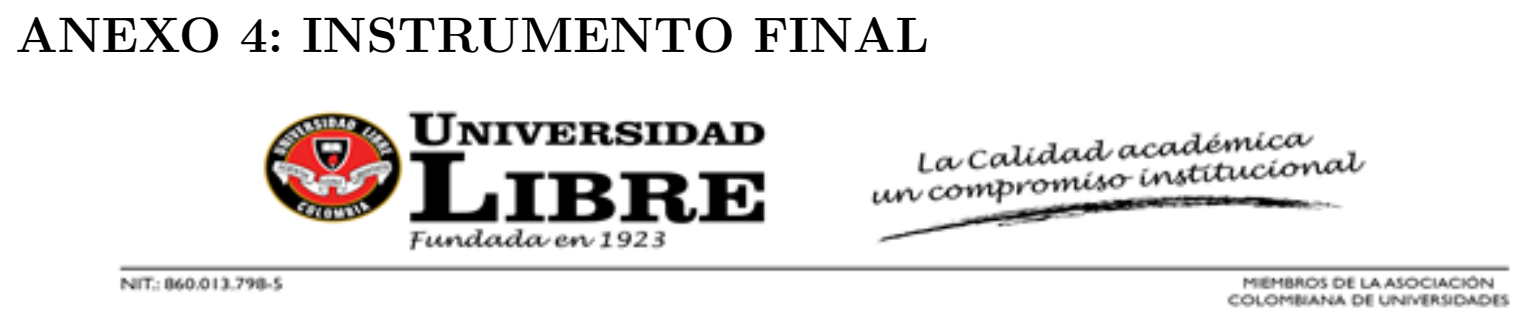

\section{INSTRUCTIVO}

Validación y aplicación de un instrumento para medir conocimientos, percepciones de riesgo y estigma sobre tuberculosis en personas privadas de la libertad

\section{FECHA: CÓDIGO PARTICIPANTE:}

Esta encuesta tiene como objetivo validar un instrumento para medir conocimientos, percepciones de riesgo y estigma que tiene las personas privadas de la libertad acerca de la tuberculosis. Los resultados que se obtengan del presente estudio servirán a otros investigadores como una escala de medida.

Después de leer cada pregunta responda según sea el caso.

\section{CONOCIMIENTO}

1. ¿Para usted qué es tuberculosis?

2. La primera vez que usted escuchó información sobre tuberculosis fue

- Dentro de la cárcel

- Fuera de la cárcel

- Nunca ha escuchado información sobre tuberculosis

3. En la cárcel usted ha recibido información sobre tuberculosis a través de: (puede marcar varias opciones)

- Personal de sanidad

- Compañero de patio

- Promotor de salud 
- Guardias del INPEC

- Visitantes o familia

- Carteleras, folletos, pendones dentro de la cárcel.

- No ha recibido información

4. ¿Qué síntomas de tuberculosis conoce usted?

5. ¿Usted cree que la tuberculosis se puede contagiar?

- Sí

- No

- No sé

6. ¿Cómo cree usted que se puede contagiar la tuberculosis en la cárcel?

7. ¿Usted cómo previene el contagio de tuberculosis?

8. ¿Usted cree que la tuberculosis tiene cura?

- Sí

- No

- No sé

PERCEPCIÓN DE RIESGO 9. ¿Usted cree que la tuberculosis puede causar la muerte?

- Sí 
- No

- No sé

10. ¿Usted cree que la tuberculosis en la cárcel se puede evitar?

- Sí

- No

- No sé

11. ¿Qué haría usted si un compañero de patio tiene tuberculosis?

\section{ESTIGMA}

12. ¿Las personas enfermas con tuberculosis tienen más limitaciones para desempeñar sus actividades diarias?

- Sí

- No

13. ¿Usted le tendría miedo a alguien que está enfermo de tuberculosis?

- Sí

- No

14. ¿Usted cree que las personas se sienten incómodas al estar cerca de alguien enfermo de tuberculosis?

- Sí

- No

15. ¿Usted cree que se debe sentir lástima por las personas enfermas de tuberculosis?

- Sí

- No

16. ¿Usted cree que las personas con tuberculosis en la cárcel, siente temor de contarle a los demás que padecen esta enfermedad? 
- Sí

- No

17. ¿Usted cree que las personas con tuberculosis dentro de la cárcel deberían estar en un patio solo para ellos?

- Sí

- No

Gracias por tu participación. 